\title{
RDUS
}

Revue de DROIT

UNIVERSITÉ DE SHERBROOKE

Titre : $\quad$ LA DÉCISION CHRISTENSEN C. ARCHEVÊQUE CATHOLIQUE

ROMAIN : LORSQUE LE TUTEUR DOIT POURSUIVRE L'AGRESSEUR

Auteur(s): Julie MCCANN

Revue: $\quad$ RDUS, 2009-2010, volume 40, numéro 1-2

Pages: $\quad 599-624$

ISSN : $\quad 0317-9656$

Éditeur : $\quad$ Université de Sherbrooke. Faculté de droit.

URI : $\quad$ http://hdl.handle.net/11143/10461

DOI : $\quad$ https://doi.org/10.17118/11143/10461 
Page vide laissée intentionnellement. 


\title{
COMMENTAIRE
}

\section{LA DÉCISION CHRISTENSEN C. ARCHEVÊQUE CATHOLIQUE ROMAIN : LORSQUE LE TUTEUR DOIT POURSUIVRE L'AGRESSEUR}

\begin{abstract}
par Julie MCCANN*
Le délai entre le moment où elle subit des actes d'agression et celui où la victime de telles agressions entame des procédures judiciaires civiles peut parfois être de quelques décennies, surtout si elle était mineure au moment des agressions. La prescription du recours de la victime d'agression sexuelle a ceci de singulier que l'extinction du droit de poursuivre peut survenir avant même que la victime n'ait été en mesure d'établir le lien entre les actes fautifs et le préjudice souffert. La question de l'impossibilité d'agir des parents, comme motif de suspension de la prescription suscite plusieurs questions liées au rôle du tuteur et à la protection de l'enfant. L'analyse de la décision Christensen c. Archevêque catholique romain de Québec nous permet de remettre en contexte la délicate question des agressions sexuelles d'enfants, à la lueur notamment de la Convention sur les droits de l'enfant.
\end{abstract}

Several decades may elapse between a sexual assault on a person and the filing of a civil action, especially when the victim was underage at the time of the assault. In this type of situation, extinctive prescription can adversely affect the victim's recourse and indeed, may extinguish the right to obtain indemnification even before the victim has realized the existence of a causal relation between the wrongful act and the injury suffered. In these cases, parents acting in their capacity as tutors may also be unable to act on behalf of their pupils. Suspension of prescription raises several issues relating to the parents' role as tutors as well as to their duty to protect their children. This comment analyses the case of Christensen v. Roman Catholic Archbishop of Québec and aims at encouraging the courts to examine the sensitive issues surrounding the sexual abuse of children in light of the Convention on the Rights of the Child.

* $\quad$ Avocate, Doctorante à l'Université McGill, Chargée de cours à l'Université Laval. L'auteure a rédigé La prescription des créances : à la croisée du droit substantiel et du droit processuel, mémoire de maîtrise, Faculté des études supérieures, Université Laval, 2007, qui a remporté le prix 2009 de l'Association des professeurs de droit du Québec pour le meilleur mémoire de maîtrise. L'auteure remercie M. Martin Thiboutot, étudiant au baccalauréat, pour son aide à la recherche. Les apports des étudiantes Isabelle Dalpé, Stephany El-Murr, Sarah Rineau-Rossi et Marie-Christine Roy ont aussi permis d'alimenter la réflexion précédant la rédaction de cet article. 


\section{SOMMAIRE}

I. L'historique des procédures judiciaires 601

II. L'irrecevabilité préliminaire fondée sur la prescription du recours 602

III. La prescription du recours fondé sur l'agression sexuelle d'une victime mineure

a) Les fondements de la prescription extinctive revisités 606

b) Le nécessaire équilibre entre les droits du débiteur (agresseur) et ceux du créancier (victime) ..................................................... 608

c) La difficile détermination du point de départ de la prescription pour les victimes d'agressions sexuelles.

IV. Les agressions sexuelles d'enfants mises en contexte

a) L'article 2904 à la lueur des droits de l'enfant .... 618

b) Les obligations du tuteur............................. 620 
Le Québec assiste depuis quelques années à une augmentation marquée du nombre de recours de victimes d'agressions sexuelles à l'encontre de leur agresseur, parfois plusieurs années après les gestes fautifs. Un exemple parmi tant d'autres est l'affaire Christensen c. Archevêque catholique romain de Québec ${ }^{1}$, où la question de l'impossibilité d'agir des parents, comme motif de suspension de la prescription, s'est posée.

\section{L'historique des procédures judiciaires}

La victime, "Shirley Christensen ", intente, en juin 2007, un recours en responsabilité civile fondé sur des allégations d'agressions sexuelles. Les actes reprochés au défendeur, l'ancien curé Paul-Henri Lachance, auraient eu lieu de manière répétée, à la fin des années 1970 et au début des années 1980; la victime était alors âgée de 6 à 8 ans. En juillet 1981, un acte plus envahissant se produit, ce qui amène la victime à se confier à ses parents. Ces derniers rencontrent les représentants de l'Archevêque catholique romain de Québec (ci-après l'" Archevêque "), lesquels affirment se charger de l'affaire et leur demandent de ne pas ébruiter l'affaire. En 2006, après avoir pris conscience des conséquences des agressions subies, la victime dépose une plainte pour attentat à la pudeur d'une personne de sexe féminin à laquelle le défendeur Lachance plaide coupable. Un recours civil en dommages est par la suite intenté en Cour supérieure. Les défendeurs, Lachance et l'Archevêque, à titre de commettants, déposent, à titre préliminaire, une requête en irrecevabilité fondée sur la prescription du recours de la demanderesse.

La décision de première instance : l'absence d'allégation de crainte comme motif de suspension de la prescription - La requête en irrecevabilité est accueillie en Cour supérieure. De l'avis du premier juge, le délai de prescription a commencé à courir au plus tard en 1983. En l'absence d'un état de crainte

1. 2010 CSC 44 [l'affaire Christensen]. 
entraînant une impossibilité d'agir, aucun motif sérieux ne permet de conclure prima facie que le délai de prescription ait été suspendu. Le recours est donc prescrit.

La décision en appel : l'absence de motif appuyant l'impossibilité d'agir des parents - La majorité des juges de la Cour d'appel rejette l'appel, mais ne fait pas siens les motifs du juge de première instance. La Cour d'appel est d'avis que le recours est prescrit. Le point de départ de la prescription est fixé à juillet 1981, moment où la victime ou ses parents auraient pu agir. La Cour rejette l'argument voulant qu'il y ait eu des préjudices distincts, préférant qualifier de manifestation graduelle l'aggravation de l'état de la victime. Les parents n'ayant pas été dans l'impossibilité d'agir, il n'y aurait pas eu de suspension de prescription.

La dissidence du juge Chamberland - Le juge Chamberland, dissident en Cour d'appel, aurait accueilli le pourvoi et rejeté la requête en irrecevabilité au motif qu'en tenant les faits pour avérés, le juge de l'irrecevabilité aurait dû faire preuve de prudence et éviter de trancher une question de fait qui aurait dû être tranchée par le juge du fond. Il est d'avis que, telles que rédigées, les allégations de la requête introductive d'instance établissent le point de départ de la prescription à l'été 2006 et que seule la preuve au procès permettrait d'en apprécier le bien-fondé.

La décision rendue par la Cour suprême - La Cour suprême a, à l'unanimité, accueilli l'appel. Dans une décision laconique, le plus haut tribunal endosse les motifs retenus par le juge Chamberland, dissident en cour d'appel.

\section{L'irrecevabilité préliminaire fondée sur la prescription du recours}

La cause dont il est question reposait essentiellement sur une question d'irrecevabilité du recours de la victime (ou de son tuteur) en application de l'article 165 (4) C.p.c : 
La décision Christensen $c$.

(2009-10) 40 R.D.U.S. Archevêque catholique romain :

603

lorsque le tuteur doit poursuivre l'agresseur

Le défendeur peut opposer l'irrecevabilité de la demande et conclure à son rejet :

[...]

4. Si la demande n'est pas fondée en droit, supposé même que les faits allégués soient vrais.

Un recours prescrit est, à sa face même, non fondé en droit et par conséquent irrecevable ${ }^{2}$. Dans le cas d'une requête en irrecevabilité, présentée à titre préliminaire au motif que l'action est non fondée en droit, la cour doit "s'interroger sur la question de savoir si l'ensemble des faits allégués dans la requête et tenus pour avérés pouvait juridiquement donner naissance au remède recherché "3. La requête en irrecevabilité pourra être accueillie à la condition que la situation de droit soit claire et évidente ${ }^{4}$. À cette étape, le juge doit :

- Tenir pour avérés les seuls faits allégués, sans toutefois tenir pour acquis la qualification juridique qui leur est donnée et prendre connaissance des pièces déposées à leur soutien ${ }^{5}$;

- Éviter de rechercher d'autres hypothèses, ou tenter d'imaginer ou deviner quels autres faits une partie aurait pu ou pourrait à l'avenir ajouter;

- Déterminer si, tenant les faits allégués, mais seulement ceux-ci, pour avérés, il peut rejeter en bloc

2. Il suffit ici de rappeler la définition de la prescription extinctive à l'effet que la prescription extinctive est " un moyen [...] d'opposer une fin de non-recevoir à une action", art. 2921 C.c.Q.

3. Gillet c. Arthur, [2005] R.J.Q. 42 (C.A.); Bourcier c. Citadelle (Compagnie d'assurances générales), [2007] R.R.A. 543 (C.A.); Fruits de Mer Gascon ltée c. Brotherton, [2000] J.Q. no 5167, REJB 2000-2162 1 (C.S.).

4. $\quad H a m p s t e a d$ (Ville de) c. Jardins Tuileries ltée, [1992] R.D.J. 163 (C.A.).

5. Denis Ferland et Benoît Emery, dir., Précis de procédure civile du Québec, 4e éd., Cowansville, Yvon Blais, 2003 aux pp. 287-288; Osmani c. École Barthélémy-Vimont, [1994] R.D.J. 581 (C.A.); O’Hearn c. Roy, [1997] R.R.A. 64 (C.A.); Creighton c. Immeubles Trans-Québec inc. [1988] 37 R.J.Q. 27 (C.A.) au par. 28; Gille c. Placements Diar inc., [1992] R.J.Q. 2746 (C.A.); Godin c. Société canadienne de la Croix-Rouge, 1993 CanLII 3881 (C.A. Qué.); Fruits de M Gascon ltée c. Brotherton, supra note 3 au par. 15 . 
la requête; et à cette fin, examiner le lien de droit entre l'ensemble des faits allégués, même si disparates ou confus, et le remède recherché;

- Faire preuve de prudence;

- N'accueillir la requête en irrecevabilité que s'il est convaincu que la demande n'est pas fondée en droit, qu'elle n'a aucune chance de succès, en supposant que tous les faits allégués soient vrais. Autrement dit, s'abstenir de mettre fin prématurément à un procès ${ }^{6}$, à moins d'être convaincu du bien-fondé de l'irrecevabilité.

Dans des situations où l'impossibilité psychologique d'agir est invoquée comme motif de suspension de la prescription, le rôle du juge statuant sur l'irrecevabilité consiste à se demander si les faits allégués à la requête introductive d'instance et les pièces produites à leur soutien :

(1) permettent de déterminer avec suffisamment de certitude quel était le point de départ de la prescription et que le délai de prescription est échu et

(2) supportent la thèse de l'impossibilité d'agir de la partie demanderesse ou de son tuteur.

La requête en irrecevabilité présentée au motif que le recours est prescrit ne sera accueillie que s'il est clair ${ }^{7}$, à la lecture des allégations de la requête et des pièces à leur soutien, que le délai pour être entendu est expiré. Par conséquent, dans les cas de prescription d'un recours basé sur une manifestation graduelle d'un préjudice, les tribunaux doivent faire preuve de circonspection au stade de l'irrecevabilité; règle générale "le moment où le préjudice s'est manifesté d'une façon appréciable

6. Hampstead (Ville de) c. Jardins Tuileries ltée, supra note 4.

7. "[L]a prescription invoquée doit apparaître de façon claire et précise sans nécessité d'interpréter avec subtilité les nuances de la jurisprudence et de la loi qui s'appliquent ", C.I.B.C. Equipment Finance Ltd. c. Transport J.M. Thibeault et Fils inc., 2002 CanLII 24900 (C.S. Qué.); Québec (Ville de) c. Constructions Bé-Con inc., 2003 CanLII 33217 (QC C.S.); (2003) R.L. 311 (C.S.) au par. 18 et 19. 
nécessite une évaluation de la preuve qui relève du juge du fond " 8 . Dans les situations où le recours semble prescrit prima facie, mais où le demandeur allègue une impossibilité d'agir ayant vraisemblablement pu interrompre la prescription, l'appréciation des faits supportant cette impossibilité en fait doit être laissée à l'appréciation du juge du fond.

Avec respect pour l'opinion du premier juge, il nous semble que dans ces situations, la victime n'a pas à démontrer, "selon la balance des inconvénients [...] qu'elle était dans l'impossibilité d'agir " 9 . Une simple déclaration à cet effet dans la requête introductive d'instance, au stade de l'irrecevabilité, devrait suffire. Comme il sera discuté plus loin dans le texte, l'impossibilité d'agir du représentant doit être examinée à la lueur du préjudice et des dommages soufferts par la victime; s'il est allégué qu'elle ne pouvait agir avant 2006, alors son représentant ne le pouvait pas non plus.

\section{La prescription du recours fondé sur l'agression sexuelle d'une victime mineure}

La décision à l'étude amène un nombre de questions particulièrement délicates compte tenu de la nature du recours. Dans un contexte où ni la jurisprudence ni la littérature ne se sont penchées sur la question, il convient (1) de revisiter les fondements de la prescription extinctive, (2) de rappeler qu'il est nécessaire de se questionner sur l'équilibre entre les droits du débiteur (agresseur) et ceux du créancier (victime) et (3) de se pencher sur la difficile détermination du point de départ de la prescription pour les victimes d'agressions sexuelles.

8. Investissements Intergem inc. c. Ultramar Canada inc., 2006 QCCA 1470 au par. 3.

9. $\quad$ S.C. c. Lachance, 2008 QCCS 2724 au par. 25. 


\section{a) Les fondements de la prescription extinctive revisités}

Historiquement, quatre principaux fondements ont servi à justifier qu'une réclamation, autrement bien fondée, puisse être considérée comme irrecevable parce que prescrite. La prescription est donc tour à tour considérée comme un outil de protection du débiteur ou une sanction de la négligence du créancier à faire valoir ses droits. Elle sert parfois de présomption de paiement et permet d'assurer la stabilité des relations juridiques, dans un souci d'ordre public ${ }^{10}$.

La prescription extinctive vient établir le droit du débiteur à la tranquillité d'esprit une fois qu'un certain délai s'est écoulé entre l'exigibilité de la dette qu'il a envers le créancier; il peut se considérer comme libéré et ne pas craindre que de trop anciennes dettes ne reviennent le hanter. Cette protection du débiteur s'accompagne aussi d'une sanction de la conduite du créancier négligent: en ne faisant pas valoir ses droits diligemment, la prescription sanctionne son inaction en rendant son recours tardif irrecevable.

Ces fondements doivent toutefois être mis en perspective : en présence d'un recours bien-fondé pour lequel le débiteur a été empêché de faire valoir ses droits, il faut éviter de privilégier la tranquillité d'esprit du débiteur au détriment des droits du créancier. C'est pourquoi nous remettons en question ce passage, abondamment cité :

[I]l faut prendre bien garde de ne point relaxer la computation des délais, de déchéance comme de procédure, au point de les rendre presque inopérants, car ces clauses servent la justice et ont pour raison d'être la protection de droits que le législateur a voulu à certaines

10. Voir à ce propos le développement portant sur les fondements de la prescription extinctive dans Julie McCann, La prescription des créances : à la croisée du droit substantiel et du droit processuel, mémoire de maîtrise, Faculté des études supérieures, Université Laval, 2007. 
La décision Christensen $c$.

(2009-10) 40 R.D.U.S. Archevêque catholique romain :

conditions privilégier, fût-ce au détriment de ceux des autres en les plaçant à l'abri des plaideurs qui se manifestent tardivement [...]. Aussi ne faudrait-il donc pas élargir outre mesure la notion de "l'impossibilité absolue en fait d'agir " que prévoit l'art. 2232 du Code civil comme fondement d'une suspension des délais ${ }^{11}$.

Cette position étant encore souvent celle des tribunaux aujourd'hui12, il importe de rappeler que depuis 1994, le législateur a choisi d'assouplir le caractère strict ${ }^{13}$ du délai et de la notion d'impossibilité d'agir en écartant le terme "absolue " qui la qualifiait alors.

Nous remarquons qu'avec le temps, les tribunaux ont privilégié une application stricte des délais de prescription extinctive alors qu'en fait, tout milite pour que l'équité ${ }^{14}$ serve de guide, surtout au stade de la requête en irrecevabilité. Une appréciation fondée sur l'équité doit avoir pour trame de fond qu'au stade de l'irrecevabilité, l'idée est de distinguer la victime " passive" de la victime qui a réellement été psychologiquement dans l'incapacité d'agir.

11. Oznaga c. Société d'exploitation des loteries et courses du Québec, [1981] 2 R.C.S. 113 au par. 31.

12. Par exemple dans la décision Catudal c. Borduas, 2006 QCCA 1090, (Requête pour autorisation de pourvoi à la Cour suprême rejetée).

13. "L'impossibilité en fait d'agir exigée par la loi demeure donc une question laissée à l'appréciation des tribunaux [...] qui devraient, eu égard au texte de l'article 2904 C.c. [...] se montrer moins stricts que sous l'ancien régime. "Jean-Louis Baudouin et Patrice Deslauriers, "La prescription " dans La responsabilité civile, Volume I - Principes généraux, 7e éd., 2007, EYB2007RES30 au par. 1-1420.

14. " [L]'équité doit présider à l'application des règles et l'individualité des cas rend parfois dangereuse une trop grande généralisation ", ibid. au par. 11418. 


\section{b) Le nécessaire équilibre entre les droits du débiteur (agresseur) et ceux du créancier (victime)}

Dans le cadre d'un recours civil, en matière de créances, le droit ne distingue pas la nature de la créance : il s'agit d'un recours en dommages-intérêts, pour compenser l'inexécution d'une obligation - de nature contractuelle ou extracontractuelle. Une nuance s'impose toutefois dans le cas de dommages exemplaires, où le caractère de l'atteinte (" illicite et intentionnel ") et la nature de l'atteinte (" un droit garanti par la charte ") seront examinés ${ }^{15}$. Une présomption existe voulant que la prescription extinctive est édictée à l'avantage du débiteur. Cette quiétude du débiteur, corollaire d'une présomption de l'abandon du droit à la réparation du créancier qui néglige de le faire valoir, ne peut être appliquée de manière stricte dans les cas d'agressions graves ${ }^{16}$. Dans ces situations, par souci d'équité, on doit tenir compte du fait que les " créances " reposent sur des événements socialement hautement répréhensibles, et même constitutifs d'infractions criminelles.

Comment les tribunaux évaluent-ils la gravité du délit d'agression sexuelle dans le contexte de recours criminels?

Il ressort de cela que la nature du délit et l'état de vulnérabilité de la victime doivent être pris en compte lorsqu'il s'agit de pondérer les droits de la victime et ceux de l'auteur du préjudice. Nous faisons nôtres les propos du juge Stevenson, alors juge à la Cour suprême :

Il faut beaucoup de courage et de force de caractère aux victimes d'abus sexuels pour révéler ces secrets personnels et ouvrir d'anciennes blessures. Si les procédures devaient être arrêtées en raison du seul temps

15. Charte des droits et libertés de la personne, L.R.Q., c. C-12, art. 49.

16. Louise Langevin, Nathalie Des Rosiers et al., "L'impossibilité psychologique d'agir et les délais de prescription : lorsque le temps compte " (2008) 42 R.J.T. 395 aux pp. 411-412; M.(K.) c. M.(H.), [1992] 3 R.C.S. 6, 31; Gauthier c. Baumont, [1998] 2 S.C.R. 3 au par. 67. 
La décision Christensen $c$.

(2009-10) 40 R.D.U.S. Archevêque catholique romain :

609

lorsque le tuteur doit poursuivre l'agresseur

écoulé entre les mauvais traitements et la mise en accusation, les victimes seraient tenues de dénoncer ces incidents avant d'être psychologiquement prêtes à assumer les conséquences de leur dénonciation. Il a été reconnu dans d'autres contextes que le retard à dénoncer les abus sexuels est une conséquence commune et prévisible dans ces cas... Si les tribunaux devaient imposer une prescription, cela signifierait que les auteurs d'abus sexuels pourraient tirer avantage de l'absence de dénonciation dont ils sont, dans bien des cas, eux-mêmes responsables. Ce n'est pas là une conséquence que nous devrions encourager ${ }^{17}$. [nos italiques]

Le fait que la victime se retrouve demanderesse dans une instance civile n'enlève rien au caractère criminel des gestes subis. En classant l'agression sexuelle parmi les infractions criminelles et en permettant aussi l'imposition de peines pour de tels délits, un message est envoyé à l'effet que "la conduite du contrevenant doit être punie parce qu'elle a porté atteinte au code des valeurs fondamentales de notre société "18; il s'agit d'une atteinte à l'ordre public. La gravité particulière de la faute commise, laquelle donnerait éventuellement lieu à l'octroi de dommages exemplaires en raison de son caractère illicite et intentionnel, milite en faveur d'une interprétation de la prescription qui ne soit pas toute à l'avantage du débiteur.

On remarque aussi que dans les cas d'agressions sexuelles commises sur des enfants, la Cour est généralement réticente à

17. R. c. L. (W.K.), [1991] 1 R.C.S., 1091, 1100 et 1101.

18. "La société doit, par l'entremise des tribunaux, communiquer sa répulsion à l'égard de certains crimes, et les peines qu'ils infligent sont le seul moyen qu'ont les tribunaux de transmettre ce message. [...] Notre droit criminel est également un système de valeurs. La peine qui exprime la réprobation de la société est uniquement le moyen par lequel ces valeurs sont communiquées à enseigner de manière positive la gamme fondamentale des valeurs communes que partagent l'ensemble des Canadiens et des Canadiennes et qui sont exprimées par le Code criminel. ", R. c. M. (C.A.), [1996] 1 R.C.S. 500 au par. 81. 
considérer que le délai19 écoulé entre les infractions commises et le prononcé de la sentence puisse être un facteur atténuant.

Le long délai écoulé entre la commission des infractions et la dénonciation n'est pas une circonstance exceptionnelle dans le cas des infractions d'ordre sexuel commises sur de jeunes enfants. Souvent, le long délai constitue une caractéristique inhérente à ce genre de crime puisque les victimes ne sont pas en mesure de se plaindre étant donné leur jeune âge, leur totale vulnérabilité et, souvent, les menaces qui les tiennent bâillonnées ${ }^{20}$.

Bien que les lois sur la prescription des actions soient " des lois destinées à assurer la tranquillité d'esprit ", les tribunaux devraient refuser de leur donner une application stricte dans des cas où la nature du recours fait échec à la protection de la tranquillité d'esprit du défendeur, par exemple dans les cas d'inceste ou d'agression sexuelle, où il serait inéquitable de favoriser la tranquillité d'esprit de l'agresseur au détriment de la victime.

\section{c) La difficile détermination du point de départ de la prescription pour les victimes d'agressions sexuelles}

Intrinsèquement liés à la naissance du droit d'action, les recours en responsabilité civile reposant sur la commission d'agressions sexuelles posent certains problèmes particuliers, compte tenu de la nature du préjudice et éventuellement des dommages causés. À partir de quel moment le droit d'action

19. La Cour d'appel a été appelée à examiner le délai de 17 ans écoulé depuis les agressions et où l'agresseur plaidait qu'il occupait un emploi stable et entretenait une relation stable à titre de circonstances atténuantes. Il soutenait aussi "qu'au terme de deux décennies, il faut laisser émerger l'oubli et la réconciliation " ce à quoi la Cour a répondu que les " caractéristiques personnelles qui s'attachent au bien-être du délinquant ne témoignent ni de la dénonciation ni de la dissuasion générale qui doit frapper les crimes sexuels commis sur des enfants en bas âge ", $R$. c. $L$. (J.J.), 1998 CanLII 12722 (C.A. Qué.).

20. Ibid. 
La décision Christensen $c$.

(2009-10) 40 R.D.U.S. Archevêque catholique romain :

lorsque le tuteur doit poursuivre l'agresseur

prend-il naissance? Poser cette question nous ramène inévitablement à se demander à quel moment la faute, le préjudice et le lien causal surviennent.

La faute - De l'avis du professeur Gardner, "la victime d'une agression sexuelle subit un préjudice corporel, puisque l'atteinte à son intégrité physique ou psychique est patente ${ }^{21}$. De cela, nous retenons donc que le moment de la (ou des fautes) est facilement identifiable et lié aux actes d'agression. La détermination du moment de la commission de la ou des fautes est ce qui semble poser le moins de problèmes.

Le préjudice - La difficulté dans la détermination du moment de la réalisation du préjudice est liée à la nature même du dommage subi : il s'agit d'un dommage, d'une conséquence invisible qui sera bien souvent latente et dont les effets néfastes pourront surgir de manière contemporaine aux agressions, ou encore plusieurs années plus tard. L'enfant qui n'a pas conscience du caractère répréhensible des actes pourra ne présenter de séquelles qu'à l'âge adulte. Sa cause d'action étant reportée d'autant ${ }^{22}$. Ainsi s'exprimait la Cour d'appel dans Christensen :

[65] Le point de départ de la prescription représente le premier moment où la personne victime d'un préjudice peut agir [...]. Il arrive que l'acte fautif produise immédiatement un préjudice; dans un tel cas, la prescription débute dès cet instant [...]. En d'autres circonstances, il se peut que le préjudice se réalise de façon graduelle. Le point de départ de la prescription est

21. Daniel Gardner, "La qualification de préjudice corporel et ses conséquences " dans Le préjudice corporel, 3e éd., 2009, EYB2009EPC3 au par. 24.

22. "La jurisprudence, plus réaliste, fait partir le délai du jour de la réalisation du préjudice, puisque c'est à ce moment seulement que les conditions juridiques du droit de poursuite se trouvent enfin réunies [...] ", Jean-Louis Baudouin et Patrice Deslauriers, supra note 13 au par. 1-1420. 
alors le moment où le préjudice se manifeste de façon appréciable $[. .$.$] .$

La manifestation appréciable du préjudice - La prescription commence dès que le préjudice apparaît pour la première fois (art. 2926 C.c.Q.). Dans le cas de préjudices corporels dont les conséquences dommageables seront échelonnées dans le temps - et ne se manifesteront pas seulement en une seule fois, mais bien graduellement - il ressort que la "première manifestation " puisse ne pas suffire à la naissance de la cause d'action. De l'avis de certains auteurs, il faudrait que "le critère de la "première fois" soit défini comme étant le moment où la demanderesse prend conscience de l'importance et de l'étendue du préjudice, même si ce dernier ne s'est pas totalement manifesté $»^{23}$.

[67] Le moment où les différents préjudices subis par l'appelante se seraient manifestés pour la première fois n'apparaît cependant pas aussi clairement des procédures.

Les dommages causés à une victime d'agressions sexuelles devraient être appréciés à la lueur des "séquelles " 24 que la faute a entrainées. Ces séquelles peuvent entrainer en divers troubles psychiques et physiques qui se manifesteront au fil de la vie de la victime; celle-ci pourra ne prendre conscience du lien entre le préjudice subi et ses conséquences qu'une fois rendue adulte ${ }^{25}$.

23. Louise Langevin, "Suspension de la prescription extinctive : à l'impossible nul n'est tenu" (1996) 56 R. du B. 185 à la p. 54 au par. 113.

24. "[L]e Tribunal ne peut retenir que le préjudice [...] existait lorsqu'il a reçu son congé de l'Hôpital [...]. Son préjudice ne se limite pas au coma profond et à la détresse respiratoire qui ne sont que les manifestations de l'injection d'une surdose de phénobarbital: ce sont les séquelles que le [...] neurologue, n'était pas encore en mesure de constater [...] qui font l'objet de la poursuite. ", Lévesque c. Centre hospitalier universitaire de Sherbrooke, 2003 CanLII 54459 (C.S. Qué.)

25. "Un événement particulier pourra également agir à titre d'élément déclencheur, qui fera prendre conscience à la victime de son préjudice et 
La décision Christensen $c$.

(2009-10) 40 R.D.U.S. Archevêque catholique romain :

Dans une instance criminelle, la Cour d'appel retient que l'" âge de la victime est un élément intimement lié à l'évaluation du rapport d'autorité qu'entretient l'agresseur avec celle-ci " et [que] dans ces circonstances les séquelles psychologiques chez la victime sont plus facilement inférées par les tribunaux $" 26$. Donc, bien que les séquelles psychologiques causées par de tels agissements puissent objectivement être prévisibles, ce n'est qu'un examen subjectif de la situation particulière de la victime qui pourra permettre de déterminer à partir de quel moment est née la cause d'action de la victime.

Le lien de causalité - L'autre problème lié à la naissance de la cause d'action réside dans le lien de causalité entre les actes fautifs et les dommages soufferts. En effet, une personne extérieure peut très bien " anticiper " que les actes fautifs puissent entraîner des séquelles, encore faut-il que la victime elle-même soit en mesure d'établir ce lien. Le lien peut être présumé fait à "partir du jour où une victime raisonnablement prudente et avertie pouvait soupçonner le lien entre le préjudice et la faute "27; c'est le critère de la "victime raisonnable placée dans les mêmes circonstances".

Comme la naissance de la cause d'action doit déterminer le début de l'écoulement du délai de prescription, il semble inéquitable d'imposer à la victime d'intenter son recours dans un délai de trois ou quatre ans après avoir acquis sa majorité, alors qu'elle n'est pas en mesure de faire le lien entre les gestes posés et le préjudice subi. "[I]l faut préalablement que la partie demanderesse soit réellement consciente du préjudice subi et de sa cause probable ${ }^{28}$. En matière de délits sexuels, le lien de

du lien existant avec la situation d'abus dont elle a été victime", Daniel Gardner, supra note 21 au par. 40.

26. William A. Schabas, Les infractions d'ordre sexuel, Cowansville, Yvon Blais, 1995 à la p. 271, cité dans R. c. R.D., 2008 QCCA 1641 au par. 54.

27. Jean-Louis Baudouin et Patrice Deslauriers, supra note 13 au par. 11420.

28. M.(K.) c. M.(H.), supra note 16. 
causalité entre les actes commis et le préjudice souffert est souvent refoulé ou tout simplement neutralisé par le syndrome du choc post-traumatique ${ }^{29}$.

En common law, la notion de possibilité raisonnable de découvrir le préjudice subi a été développée ${ }^{30}$. Elle sous-tend un cheminement intellectuel entrepris par la victime lui permettant de relier les dommages subis aux actes incestueux, et donc de faire naître la cause d'action. La découverte du préjudice subi n'est donc possible qu'une fois que la victime se trouve en mesure de lier les éléments de faute et de préjudice. La Cour suprême a établi une présomption selon laquelle " c'est seulement en suivant une forme quelconque de psychothérapie que certaines victimes d'inceste découvrent le lien nécessaire entre les préjudices qu'elles ont subis et le mal qu'on leur a fait (et ainsi leur cause d'action) "31. Cette présomption facilite la preuve de la découverte du préjudice subi en la reliant à l'aide thérapeutique reçue : elle peut être réfutée par une preuve à l'effet contraire, soit que la victime était consciente du préjudice subi et de son caractère répréhensible à un moment antérieur. Cette présomption semble tranquillement se frayer un chemin en droit québécois ${ }^{32}$.

On peut aussi raisonnablement s'attendre à ce que soient appliqués ces mêmes critères, et même de manière assouplie,

29. Louise Langevin, Nathalie Des Rosiers et al., supra note 16 à la p. 415 .

30. En droit américain, on observe une tendance similaire, soit la règle de la découverte tardive, voir M.(K.) c. M.(H.), supra note 16.

31. Ibid.

32. Dans A c. B, 2007 QCCS 5, EYB 2007-112340 (C.S.), J.E. 2007-288 (C.S.), [2007] R.R.A. 172 (C.S.) au par. 61, La cour invoque la décision M.(K.) c. M.(H.), supra note 16 , qui pose que "On peut présumer que cette prise de conscience a lieu au moment où la victime reçoit une certaine forme d'aide thérapeutique, que ce soit d'un professionnel ou de la collectivité en général. J'en suis venu à cette conclusion après avoir étudié les témoignages d'experts en l'espèce et la jurisprudence américaine qui s'est attaquée à ce problème au cours de la dernière décennie. Il va sans dire que cette présomption sera écartée si la preuve révèle que la victime a découvert le préjudice et sa cause probable à un autre moment " [nos italiques]. 
La décision Christensen $c$.

(2009-10) 40 R.D.U.S. Archevêque catholique romain :

lorsqu'un représentant légal est tenu d'entreprendre un recours ayant pour fondement un préjudice qu'il ne subit pas lui-même.

La suspension de la prescription : l'impossibilité d'agir du mineur ou du tuteur? - On reconnaît au détenteur d'un droit une suspension du délai pour agir en justice lorsqu'il se trouve dans l'" impossibilité d'agir " ou encore lorsqu'il n'est pas en mesure de connaître les éléments essentiels de sa réclamation. Il s'agit donc soit du jour où la partie a effectivement découvert les éléments donnant ouverture au droit qu'elle réclame ou à l'identité de la personne responsable ${ }^{33}$. De manière à ce que la preuve de la connaissance ne puisse pas couvrir l'ignorance de la partie qui aurait été négligente, le délai de prescription commence à compter du moment où une "personne raisonnable possédant les mêmes capacités et se trouvant dans la même situation que le titulaire du droit de réclamation aurait dû apprendre les faits "34 ayant donné naissance au droit d'action.

L'article 2904 C.c.Q. dispose que :

La prescription ne court pas contre les personnes qui sont dans l'impossibilité en fait d'agir soit par elles-mêmes, soit en se faisant représenter par d'autres.

L'impossibilité d'agir d'une personne ou de son représentant entraîne donc une suspension de la prescription. Dans le cas d'une personne incapable, tel un mineur ou un majeur inapte, l'impossibilité d'agir devra être appréciée à la lueur de la situation du représentant. En doctrine et en jurisprudence, il semble que

33. On considère que les faits sont découverts au jour où le titulaire apprend les faits essentiels, soit la survenance des préjudices, pertes ou dommages, les actes ou omissions les ayant causé, ou l'identité de la personne responsables de ceux-ci, Loi de 2002 sur la Prescription des actions, L.O. 2002, c. 24, ann. B, art. 5(1); "Unless otherwise provided in this Act, no proceedings shall be commenced with respect to a claim after two years from the day on which the claim is discovered. ", Limitations Act, S.S. 2004, c. L-16.1, art. 5.

34. Loi de 2002 sur la Prescription des actions, Ibid., art. 5(1) b). 
deux thèses s'affrontent quant à la portée de l'article 2904 C.c.Q. : d'une part, certains prétendent que le mineur et son représentant doivent être dans l'impossibilité d'agir pour que la prescription soit suspendue alors que d'autres soutiennent que la seule impossibilité d'agir du mineur ou de son représentant ont cet effet. À cet effet, nous tenons à rappeler que le mineur est frappé d'une incapacité juridique générale, sauf quelques exceptions : il n'est pas dans l'impossibilité d'agir, il en est tout simplement incapable. Par ailleurs, l'impossibilité d'agir du représentant est intimement liée à celle du représenté : la naissance du droit d'action doit être appréciée du point de vue de la victime. S'il est plausible que la victime elle-même ait été dans l'impossibilité d'établir le lien entre les gestes posés et le préjudice subi, comment peut-on prétendre que le représentant aurait pu (ou dû) entreprendre un recours plus tôt? L'impossibilité d'agir de la victime se transmet au représentant.

L'incapacité d'agir et la diligence raisonnable - Le défaut d'intenter un recours dans les délais prescrits peut être interprété comme un manque de diligence de la part du créancier de l'obligation de réparer. Cependant, la diligence implique et repose sur la présomption qu'une personne est au courant des droits qu'elle possède. Par exemple, les personnes frappées d'une incapacité juridique sont présumées ignorer leurs droits - et donc les recours dont elles disposent; il serait injuste de s'attendre à ce qu'elles fassent preuve de diligence, à la manière d'une personne entièrement apte ${ }^{35}$. Par ailleurs, prétendre que les victimes d'agression sexuelle qui tardent à faire valoir leurs droits font preuve d'un manque de diligence traduit une méconnaissance des conséquences psychologiques causées par un tel délit.

La déclaration de culpabilité : aveu judiciaire équivalent à reconnaissance de dette ? $^{\mathbf{3 6}}$ - Il est prévu au Code civil que la reconnaissance de dette a un effet interruptif de la prescription

35. Murphy c. Welsh; Stoddard c. Watson, [1993] 2 R.C.S. 1069.

36. Voir le développement à ce sujet dans : J. McCann, supra note 10. 
(art. 2898 C.c.Q.) ${ }^{37}$; après la reconnaissance de dette, peu importe que le délai de prescription soit échu, celui-ci recommencera à courir par le même laps de temps (art. 2903 C.c.Q.). Celui qui pouvait bénéficier de la prescription perd le bénéfice du temps écoulé s'il reconnaît - même implicitement - le bien-fondé du droit de son créancier. Il s'agit donc d'un aveu fait par le débiteur que la dette est due38. Rappelons que "[c]ette reconnaissance n'est soumise à aucune formalité impérative de la loi. Elle peut être expresse ou tacite [...]. Pour être valable, elle doit cependant montrer clairement la volonté du débiteur de reconnaître sa dette $" 39$.

L'intimé Lachance a enregistré un plaidoyer de culpabilité à une accusation criminelle d'attentat à la pudeur: bien qu'une condamnation criminelle ne puisse tenir le civil en l'état, il n'en demeure pas moins qu'il y a une reconnaissance judiciaire de l'infraction et donc, du préjudice causé. Peut-on inférer qu'il s'agit ici d'une reconnaissance de dette? Il nous semble que répondre par l'affirmative ne ferait qu'écarter l'obstacle de la prescription pour permettre qu'une preuve quant aux actes reprochés puisse être administrée.

\section{Les agressions sexuelles d'enfants mises en contexte}

On ne peut passer sous silence le contexte social plus large dans lequel s'inscrit la problématique de l'agression sexuelle d'enfants en bas âge. L'affaire Christensen porte sur des actes d'agression commis à la fin des années 1970 et au début des années 1980. Pour évaluer la situation, on doit s'attarder à

37. Deschamps c. Banque de Nouvelle-Écosse, [1989] R.D.J. 456 (C.A.); Richer c. Gironne, [1999] R.L. 498; Taillefer c. Services Immobiliers Syst.M.A. Réal inc., [1991] R.J.Q. 1788; Letarte c. Prévoyants du Canada, [1974] R.P. 428 et Caisse Populaire St-Conrad d'Anjou c. Stadler, J.E. 971374. Et Dumoulin c. Spiegel, 2003 CanLII 2878 (QC C.Q.).

38. Jean-Louis Baudouin et Pierre-Gabriel Jobin, Les obligations, $5^{\mathrm{e}}$ éd., Cowansville, Yvon Blais, 1998 à la p. 799.

39. Jean-Louis Baudouin et Patrice Deslauriers, supra note 13 au par.11434. 
l'intérêt marqué et à l'évolution de la protection des droits de l'enfant et remettre les événements dans le contexte social de l'époque pour examiner le comportement des parents.

\section{a) L'article 2904 C.c.Q. à la lueur des droits de l'enfant}

Un des éléments déterminants de l'affaire Christensen concerne l'âge de la victime au moment de la commission des actes. Elle était âgée de 6 à 8 ans, et donc, avait à peine l'âge de raison. Peu importe que ce soit cette personne maintenant devenue adulte qui intente le recours : la faute a été commise à l'égard d'une enfant.

À cet âge, l'enfant est frappé d'une incapacité juridique ${ }^{40}$. Sa capacité de jouissance est cependant entière et on en fait une catégorie de personnes auxquelles une protection additionnelle doit être conférée. "L'âge de la minorité constitue juridiquement un "avantage" puisqu'il implique normalement une protection accrue $" 41$.

En droit civil québécois, il est reconnu que toutes les décisions concernant l'enfant doivent être prises dans son intérêt (art. 33 C.c.Q.). Cependant, cette primauté du droit de l'enfant est relativement récente. Ce n'est que depuis les années 1980 que les enfants sont réellement sujets de droit dont l'intérêt a acquis un statut prépondérant ${ }^{42}$.

40. "Les personnes deviennent pleinement capables d'exercer tous leurs droits civils lorsqu'elles deviennent majeures. La loi fixe l'âge de la majorité à 18 ans (art. 153 C.c.Q.). Cette ligne de démarcation entre minorité et majorité implique que, du jour au lendemain, le mineur perd le bénéfice des mécanismes de protection de la loi ", Édith Deleury et Dominique Goubau, "La protection civile des mineurs " dans Le droit des personnes physiques, 4e éd., Cowansville, Yvon Blais, 2008, EYB2008DPP16 au par. 470 [nos italiques].

41. Ibid.

42. "La notion de l'intérêt de l'enfant [...] a été édictée pour la première fois par le législateur, comme règle générale de protection des enfants, en 1980 [...]", ibid. 
La décision Christensen $c$.

(2009-10) 40 R.D.U.S. Archevêque catholique romain :

lorsque le tuteur doit poursuivre l'agresseur

Ainsi a été érigé le système de détermination de la peine pour des infractions commises sur des enfants au motif " [qu'] en regard des infractions contre les enfants [...] la protection des enfants représente l'une des valeurs essentielles des sociétés organisées [...]".

En tant qu'État signataire de la Convention sur les droits de l'enfant, le Canada a fait de la protection des droits de l'enfant un enjeu central. Dans les cas d'agressions faites à l'égard d'enfants, la législation doit s'assurer de protéger la personne de l'enfant ${ }^{43}$; le droit à la réparation fait aussi partie des préoccupations.

Le droit des enfants à la réparation - Lorsque la victime est un enfant, la Convention accorde implicitement le droit à la réparation. Ce droit doit se traduire non seulement par le droit d'être représenté pour faire valoir ses droits, mais aussi par une procédure et des mesures compensatoires qui sont proportionnelles aux préjudices soufferts ${ }^{44}$. Faire coïncider le point de départ de la prescription avec le moment où l'acte fautif est commis peut avoir un double effet indésirable. D'une part, l'enfant devrait subir, parfois de manière prématurée, un recours civil où, en tant que témoin principal, il lui incomberait de venir faire la preuve de ses dommages. D'autre part, cela équivaudrait à lui nier un droit reconnu aux victimes adultes, soit le bénéfice de la suspension de la prescription pour impossibilité psychologique

43. "1. Les États parties prennent toutes les mesures [...] appropriées pour protéger l'enfant contre toutes formes de violence, [...] y compris la violence sexuelle, pendant qu'il est sous la garde de ses parents ou de l'un d'eux, de son ou ses représentants légaux ou de toute autre personne à qui il est confié. ", art. 19 de la Convention.

44. "Legislation concerning the right to a remedy should be reviewed with a view [...]. Ensuring that procedural norms concerning the representation of children in such proceedings, and safeguards concerning the use and control of compensation awarded to children, protect the best interests of the child ", UNICEF, Child Protection: A Handbook for Parlementarian, en ligne : <http://www.unicef.org/french/crc/files/Guide_Enfants_OK.pdf> à la p. 155 . 
d'agir ${ }^{45}$, impossibilité liée à la nature des dommages soufferts. Dans tous les cas, on doit tenir compte du fait que les questions mettant en jeu les intérêts d'un enfant font appel à un nombre de notions et d'expertises qui transcendent l'application d'un droit trop strict 46 .

\section{b) Les obligations du tuteur}

La Cour d'appel retenait principalement que les parents auraient dû intenter un recours civil, entre 1981 et 1984, au nom de leur fille :

[102] Contrairement à d'autres victimes, l'appelante a rapporté à ses parents ce qui lui était arrivé. On ne saurait donc prétendre que les parents n'avaient pas connaissance des fautes de l'intimé Lachance.

[105] Selon ce qu'il appert du rapport d'expertise [1]es parents ont consulté parce que l'appelante présentait certains troubles de comportement.

Essentiellement, on infère des faits que d'une part, les parents étaient au courant des actes fautifs et d'autre part, que leur comportement révèle qu'ils avaient aussi connaissance du préjudice souffert par leur fille et qu'ils avaient en mains toute

45. "The right of child victims to seek reparations for injuries suffered is important for several reasons. First, like any other victims, children have a right to be compensated for the moral, physical and psychological injuries caused by violation of their rights. Second, holding the perpetrators responsible economically can be an effective deterrent, especially where corporations or public or private agencies are involved in the violation. Third, compensation of the victims can help facilitate social reintegration. ", ibid. à la p.152.

46. " [L]'intérêt d'un enfant est tributaire d'un très grand nombre de facteurs difficiles à évaluer [...]. Ici, le droit ne suffit plus. En effet, il faut faire appel à des critères non juridiques, relevant essentiellement des sciences humaines [...]. C'est pourquoi les tribunaux recourent aux spécialistes de l'enfance: médecins, éducateurs, travailleurs sociaux, psychologues, psychiatres", Édith Deleury et Dominique Goubau, "Le respect des droits de l'enfant " dans Le droit des personnes physiques, 4e éd., 2008, EYB2008DPP19. 
La décision Christensen $c$.

(2009-10) 40 R.D.U.S. Archevêque catholique romain :

lorsque le tuteur doit poursuivre l'agresseur

l'information pour agir en justice. Cette affirmation doit cependant être nuancée, à la lueur du contexte historique.

Un bref survol de la situation des recours civils intentés à la suite de la commission d'actes à caractère sexuel est fort révélateur. La banque de données SOQUIJ répertorie 15426 décisions rendues avant le 31 décembre 1982. De toutes celles-ci, il ressort qu'une seule décision ${ }^{47}$, rendue en 1978 , porte sur une question liée à une agression sexuelle dans le contexte d'un procès civil.

Le problème de l'abus sexuel a toujours été présent dans la société, mais avant l'adoption de la Loi concernant la protection des enfants soumis à des mauvais traitements (L.Q. 1974, c. 59) et de la Loi sur la protection de la jeunesse (L.Q. 1977, c. 20), les centres de services sociaux de cette époque avaient peu rencontré cette réalité. L'abus sexuel était alors traité comme un problème familial et le recours à la justice, rarement utilisé ${ }^{48}$.

À l'époque où les gestes reprochés se sont déroulés, aucune sensibilisation n'était faite quant aux actes à caractère sexuel. Aussi, est-il utile de se reporter au début des années 1980, pour rappeler qu'à cette époque la femme mariée ne pouvait

47. Labonté c. Bélanger, 200-05-005134-77, 1978-01-31, AZ-78022021, J.E. 78-119, (C.S. Qué.), juge André.

48. "En 1979, l'obligation de signaler les situations d'abus sexuel provoque une réflexion dans la société québécoise, facilitant la mise en place de nouveaux modes d'intervention dans les divers réseaux et permettant aux acteurs visés d'avoir une vision globale de l'ampleur du problème. En janvier 1988, la réforme législative du Parlement fédéral relative aux infractions à caractère sexuel commises à l'égard des enfants entre en vigueur. En février 1988, [divers intervenants] adoptent une position commune pour élaborer un mécanisme de concertation sociojudiciaire en matière d'abus sexuels envers les enfants. ", Ministère de la santé et des services sociaux, Entente multisectorielle relative aux enfants victimes d'abus sexuels, de mauvais traitements physiques ou d'une absence de soins menaçant leur santé physique, 2001, en ligne : <http://publications.msss.gouv.qc.ca/acrobat/f/documentation/2000/0 0-807/00-807-04.pdf> à la p. 8. 
aucunement être victime de viol de la part de son époux ${ }^{49}$ - ou du moins ne criminalisait pas le fait pour un homme d'avoir une relation sexuelle avec sa femme, quelles que soient les circonstances et peu importe qu'elle soit consentante ou non...

Cette absence de conscientisation sociale généralisée 50 doit être prise en compte lorsque la " possibilité d'agir " des parents est évaluée. Ils ont agi dans les limites de ce que la société permettait à cette époque ${ }^{51}$, et au-delà, considérant qu'il s'agissait d'un représentant de l'Église catholique ${ }^{52}$. Ils étaient aussi justifiés de s'en remettre à la "justice " de l'Église, car même les tribunaux étaient réticents à sanctionner les actes de membres du clergé53.

49. Renée Collette-Carrière, "La victimologie et le viol, un discours complice " (1980) Criminologie 13:1 à la p. 66.

50. La lecture d'un document produit par Santé Canada en 1997 est particulièrement révélatrice quant au caractère méconnu ou tabou de l'agression sexuelle d'enfants lorsqu'on y affirme qu'à cette époque - pas si lointaine - il y avait " peu de statistiques nationales sur l'agression sexuelle d'enfants au Canada. ", Santé Canada, "L'agression sexuelle d'enfants ", Renseignements du Centre national d'information sur la violence dans la famille, 1997, en ligne: <http://dsppsd.pwgsc.gc.ca/Collection/H72-22-2-1997F.pdf $>$.

51. "[E]xaminée de plus près, la preuve porte à conclure que les actes innommables dont ces jeunes enfants ont été victimes ne pouvaient tout simplement pas être nommés : à l'époque, le plus souvent, on n'en parlait pas. Pour mes contemporains, les mesures prises étaient nettement insuffisantes et le milieu de vie, peu sûr. Toutefois, suivant les normes de l'époque, la connaissance présumée d'un risque prévisible que les enfants soient sexuellement agressés n’a pas été établie. ", Blackwater c. Plint, [2005] 3 R.C.S. 3.

52. Ringuette c. Ringuette, [2003] R.R.A. 602 (C.S.) au par. 84; Code de droit canonique (1 $\mathrm{er}$ juin 2010), en ligne : <www.vatican.va/arhive/FRA0037/ _INDEX.HTM>.

53. Guy Laperrière, "L'histoire religieuse du Québec: principaux courants, 1978-1988 ", Revue d'histoire de l'Amérique française 42:4 (1989) aux pp. 563-578. 
La décision Christensen $c$.

(2009-10) 40 R.D.U.S. Archevêque catholique romain :

623

lorsque le tuteur doit poursuivre l'agresseur

\section{CONCLUSION}

L'analyse de l'évolution des lois relatives à la prescription ailleurs au Canada nous permet de constater que celles-ci ont dû être amendées pour s'adapter aux situations de recours civils fondés sur des agressions sexuelles; dans plusieurs cas, la prescription ne court pas contre les victimes ${ }^{54}$. En ce qui a trait à la situation prévalant au Québec, nous abondons dans le sens des professeures Langevin et Des Rosiers, dont l'opinion est à l'effet que les tribunaux québécois disposent déjà des outils nécessaires à une interprétation de l'impossibilité d'agir plus sensible aux victimes d'agressions sexuelles ${ }^{55}$. Cette approche plus conciliante se dégage déjà de la jurisprudence entourant l'article 11 de la Loi sur l'indemnisation des victimes d'actes criminels ${ }^{56}$, laquelle accorde un délai d'un an à la victime pour se prévaloir des indemnités prévues à la loi; cependant, le Tribunal administratif du Québec, chargé de la révision des décisions, s'est montré attentif aux cas d'agressions sexuelles 57 en appliquant notamment

54. Certaines lois prévoient ces situations en suspendant indéfiniment le cours de la prescription en faveur des victimes d'agressions sexuelles dans des circonstances où une relation de confiance ou de dépendance existait entre la victime et l'agresseur, Limitations Act, S.S. 2004, c. L16.1; "Le délai de prescription créé par l'article 4 ne court pas dans le cas d'une réclamation fondée sur des voies de faits ou une agression sexuelle pendant toute période au cours de laquelle le titulaire du droit de réclamation est dans l'incapacité d'introduire l'instance en raison de son état physique, mental ou psychologique. ", Loi de 2002 sur la Prescription des actions, supra note 33, art. 10 (1); etc.

55. Louise Langevin, supra note 23; Nathalie Des Rosiers et Louise Langevin, L'indemnisation des victimes de violence sexuelle et conjugale, Cowansville, Yvon Blais, 1998 à la p. 67.

56. L.R.Q. c. I-6.

57. Voir notamment C.M. c. Québec (P.G.), 2008 QCTAQ 10463; A.M. c. Québec (P.G.), 2009 QCTAQ 07105; A.M. c. Québec (Procureur général) 2009 QCTAQ 03487; Louise Langevin, "La Loi sur l'indemnisation des victimes d'actes criminels du Québec : lorsque le temps court contre les victimes de violence sexuelle intrafamiliale " (2007) 48 Cahiers de Droit 681 à la p. 697. 
le principe de la présomption de connaissance, développé par la Cour suprême 58 . 